VOL. 59 (1999) [495-508]

\title{
AN EULER-TYPE VOLUME IDENTITY
}

\author{
Kevin Callahan and Kathy HanN
}

In this paper we present an elementary proof of a congruence by subtraction relation. In order to prove congruence by subtraction, we produce a dissection relating equal sub-polytopes. An immediate consequence of this relation is an Euler-type volume identity in $\mathbb{R}^{3}$ which appeared in the Unsolved Problems section of the December 1996 MAA Monthly.

This Euler-type volume identity relates the volumes of subsets of a polytope called wedges that correspond to its faces, edges, and vertices. A wedge consists of the inward normal chords of the polytope emanating from a face, vertex, or edge. This identity is stated in the theorem below.

Euler Volume Theorem. For any three dimensional convex polytope $P$

$$
\begin{gathered}
\sum \text { volume of the face wedges }+\sum \text { volume of the vertex wedges } \\
=\text { twice the volume of } P+\sum \text { volume of the edge wedges. }
\end{gathered}
$$

This identity follows immediately from

$$
\sum \text { face wedges }+\sum \text { vertex wedges } \cong 2 P+\sum \text { edge wedges. }
$$

\section{INTRODUCTION}

In this paper we present an elementary proof of a congruence by subtraction relation. In order to prove congruence by subtraction, we produce a dissection relating equal subpolytopes. An immediate consequence of this relation is an Euler-type volume identity in $\mathbb{R}^{3}$ which appeared in [2]. A proof of this volume identity was published in [3], though it used powerful tools from integral geometry, such as Federer's Area Formula. An elementary result such as this deserves an elementary proof, which is what we provide in this paper.

This Euler-type volume identity relates the volumes of subsets of a polytope called wedges that correspond to its faces, edges, and vertices. A wedge consists of the inward normal chords of the polytope emanating from a face, vertex, or edge. There is an elementary geometric argument, see [3], which shows that for a polygon, the sum of the

Received 17th December, 1998

Copyright Clearance Centre, Inc. Serial-fee code: 0004-9729/99 \$A2.00+0.00. 
areas of the wedges corresponding to edges equals the sum of the areas of the wedges corresponding to vertices. This analog of Euler's identity generalises to $\mathbb{R}^{n}$, the alternating sum is equal to $1-(-1)^{n}$ times the volume of the polytope. We call this the Euler Volume Theorem which is stated below for $\mathbb{R}^{3}$.

Euler Volume Theorem. For any three dimensional convex polytope $P$

$$
\begin{gathered}
\sum \text { volume of the face wedges }+\sum \text { volume of the vertex wedges } \\
=\text { twice the volume of } P+\sum \text { volume of the edge wedges. }
\end{gathered}
$$

This identity follows immediately from

$$
\sum \text { face wedges }+\sum \text { vertex wedges } \cong 2 P+\sum \text { edge wedges. }
$$

NOtation AND Definitions. Let $P$ be a polytope in $\mathbb{R}^{3}$. First we'll give the basic definitions. The definitions of $\stackrel{ \pm}{\cong}$ and $\cong$ can be found in Eves [1].

Definition. A normal to $P$ at a point $x$ in the boundary of $P, \partial P$, is a ray with endpoint at $x$, perpendicular to a support plane $H$ of $P$ at $x$, and contained in the halfspace bounded by $H$ which contains $P$.

DEFINITION. For each point $x \in \partial P$, the normal chords corresponding to $x$ are the chords of $P$ that lie in a normal to $P$ which emanates from $x$.

Definition. Given an $i$-dimensional face $G$ of $P$, the wedge, $W G$ associated with $G$ is the subset of $P$ containing all the normal chords of $P$ which are also normal to the affine hull of $G$, aff $(G)$.

Pictured below is a dissection of the polytope $Q$, a skewed truncated quadrilateralbased pyramid. Polytope $Q$ will be used as an example throughout this paper. The sub-polytope labelled 1 is a face wedge. The complement of this face wedge is the union of polytopes 2, 3, and 4. This complement will be used in the proof of the Euler-type Identity. Examples of edge and vertex wedges can be found in Figures 3 and 4 in Section 3.

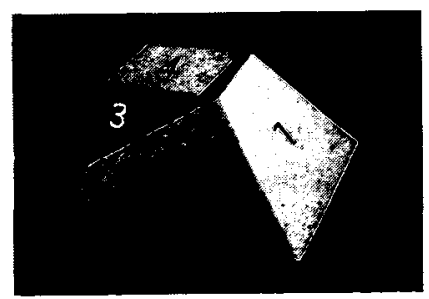

Figure 1. A Face Wedge of polytope $Q$

Before we discuss the proof, we give an elementary example of the Euler Volume Identity. Consider $P$ a cube. All of the vertex, edge and face wedges of $P$ are the entire cube. The 
reader may verify that the Euler Volume Theorem follows trivially from the classic Euler identity.

In Section 2 we prove that the Euler Volume Theorem, EVT, follows from the Wedge Complement Theorem, WCT. The WCT states that given a convex polytope $P$ in $\mathbb{R}^{3}$, the sum of the face wedge complements plus the sum of the vertex wedge complements is congruent by addition to the sum of the edge wedge complements. The remainder of the paper is dedicated to proving the WCT. In Section 3, the wedge complements will be dissected into "molecules". In Section 4, we prove that these molecules partition the wedge complements. Finally, in Section 5, a correspondence is established between the molecules, which is used to prove the WCT.

\section{The equivalence of the Euler Volume Theorem and the Wedge COMPLEMENT TheOREM}

In this section, we prove that the Euler Volume Theorem follows from the Wedge Complement Theorem which we state below.

Given a three dimensional convex polytope $P$, let $v$, e and $f$ be the number of vertices, edges and faces of $P$. Let $W V_{i}, W E_{i}$ and $W F_{i}$, be the $i^{\text {th }}$ vertex, edge and face wedge of $P$.

Wedge Complement Theorem. Given a convex polytope $P$ in $\mathbb{R}^{3}$ the sum of the face wedge complements plus the sum of the vertex wedge complements is congruent by addition to the sum of the edge wedge complements,

$$
\sum_{i=1}^{f}\left(P-W F_{i}\right)+\sum_{i=1}^{v}\left(P-W V_{i}\right) \stackrel{ \pm}{\cong} \sum_{i=1}^{e}\left(P-W E_{i}\right) .
$$

With this notation, we restate the Euler Volume Theorem and prove that it follows from the Wedge Complement Theorem.

Euler Volume Theorem. Given a convex polytope $P$ in $\mathbb{R}^{3}$ the sum of the volumes of the face wedges plus the sum of the volumes of the vertex wedges equals twice the volume of the polytope plus the sum of the volumes of the edge wedges,

$$
\sum_{i=1}^{f} \operatorname{Vol}\left(W F_{i}\right)+\sum_{i=1}^{v} \operatorname{Vol}\left(W V_{i}\right)=2 \operatorname{Vol}(P)+\sum_{i=1}^{e} \operatorname{Vol}\left(W E_{i}\right)
$$

This identity follows immediately from

$$
\sum_{i=1}^{f} W F_{i}+\sum_{i=1}^{v} W V_{i} \cong 2 P+\sum_{i=1}^{e} W E_{i}
$$

Note that the volume result is independent of measure (Euclidean or otherwise). 
PROOF THAT THE WCT IMPLIES THE EVT: We first show that the congruence by addition given in the Wedge Complement Theorem leads to the congruence by subtraction stated in the Euler Volume Theorem. The volume result follows directly from the congruence result. Using the Wedge Complement Theorem, we have

$$
\sum_{i=1}^{f}\left(P-W F_{i}\right)+\sum_{i=1}^{v}\left(P-W V_{i}\right) \stackrel{ \pm}{\cong} \sum_{i=1}^{e}\left(P-W E_{i}\right),
$$

thus there are subpolytopes $Q_{1}, \ldots, Q_{n}$ and $R_{1}, \ldots, R_{n}$ such that

$$
\begin{aligned}
\sum_{i=1}^{f}\left(P-W F_{i}\right)+\sum_{i=1}^{v}\left(P-W V_{i}\right) & =Q_{1}+\ldots+Q_{n}, \\
\sum_{i=1}^{e}\left(P-W E_{i}\right) & =R_{1}+\ldots+R_{n} \text { and } \\
Q_{i} & \cong R_{i} \text { for } i=1, \ldots, n .
\end{aligned}
$$

Therefore,

$$
\begin{aligned}
\sum_{i=1}^{f} W F_{i}+\sum_{i=1}^{v} W & V_{i}+Q_{1}+\ldots+Q_{n} \\
& =\sum_{i=1}^{f} W F_{i}+\sum_{i=1}^{v} W V_{i}+\sum_{i=1}^{f}\left(P-W F_{i}\right)+\sum_{i=1}^{v}\left(P-W V_{i}\right) \\
& =(f+v) P \\
& =(2+e) P \\
& =2 P+\sum_{i=1}^{e} W E_{i}+\sum_{i=1}^{e}\left(P-W E_{i}\right) \\
& =2 P+\sum_{i=1}^{e} W E_{i}+R_{1}+\ldots+R_{n} .
\end{aligned}
$$

Thus,

$$
\sum_{i=1}^{f} W F_{i}+\sum_{i=1}^{v} W V_{i} \cong 2 P+\sum_{i=1}^{e} W E_{i}
$$

and

$$
\sum_{i=1}^{f} \operatorname{Vol}\left(W F_{i}\right)+\sum_{i=1}^{v} \operatorname{Vol}\left(W V_{i}\right)=2 \operatorname{Vol}(P)+\sum_{i=1}^{e} \operatorname{Vol}\left(W E_{i}\right)
$$

Using the same argument, one can prove that the Wedge Complement Theorem follows from the Euler Volume Theorem and thus they are equivalent. The remainder of this paper establishes the Wedge Complement Theorem. We begin by defining terms. 


\section{Definitions}

In this section, we establish notation, give definitions and prove that the vertex, edge and face wedges can be represented using intersections of half-spaces. We also use these half-spaces in order to partition the wedge complements into "molecules". We begin by establishing notation for the faces, edges and vertices.

Given a polytope $P$, with $n$ faces, label the faces $F_{1}, \ldots, F_{n}$ in any fashion. The face labels induce a labeling on the edges and vertices. Edge $E_{i j}$ is contained in faces $F_{i}$ and $F_{j}$. Vertex $V_{*}, *=\left\{i_{1}, i_{2}, \ldots, i_{s}\right\}$, is contained in $F_{i_{t}}, t=1, \ldots, s$ where $F_{i_{1}}, \ldots, F_{i_{s}}$ are all of the faces containing $V_{*}$. Let $f_{0}(R), f_{1}(R)$ and $f_{2}(R)$ be the number of vertices, edges and faces of any polytope $R$. Three faces, $F_{1}, F_{2}$ and $F_{3}$, of the polytope $Q$ are labelled below. This induces the labeling $E_{12}$ and $V_{\{1,2,3\}}$ as pictured. Note that with this labeling, the face wedge found in Figure 1 is $W F_{1}$.

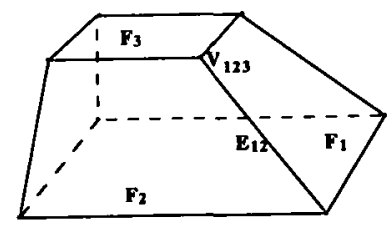

Figure 2. Some labels on the polytope $Q$

We now define two different types of cutting hyperplanes which will be used to give an alternate representation of the wedges and also to define the molecules.

Definition. Given an edge $E_{i j}$ and face $F_{i}$, the cutting hyperplane, $H_{i j}$, is the hyperplane containing this edge and perpendicular to this face.

DEFINITION. Given an edge $E_{i j}$ and one of its vertices $V_{*_{1}}$, the cutting hyperplane, $C_{i j *_{1}}$, is the hyperplane containing this vertex and perpendicular to this edge.

Definition. $H_{i j}^{+}\left(H_{i j}^{-}\right)$is the half-space containing (not containing) the face $F_{i}$ which is bounded by the hyperplane $H_{i j}$.

Definition. $C_{i j^{*}}^{+}\left(C_{i j_{*}}^{-}\right)$is the half-space containing (not containing) the edge $E_{i j}$ which is bounded by the hyperplane $C_{i j *}$.

Note that throughout this paper, the order of indices is only relevant for the cutting hyperplane $H_{i j} . H_{i j}$ is orthogonal to face $F_{i}$ whereas $H_{j i}$ is orthogonal to face $F_{j}$. Also, all half-spaces are assumed to be closed. Next we use these cutting hyperplanes to give an alternate representation of the wedges.

WEDge REPREsEntation THEOREM.

1. $W F_{i}=P \cap\left(\bigcap_{s=1}^{f_{1}\left(F_{i}\right)} H_{i s}^{+}\right)$.

2. $W E_{i j}=P \cap H_{i j}^{+} \cap H_{j i}^{+} \cap C_{i j *_{1}}^{+} \cap C_{i j *_{2}}^{+}$where $v_{*_{1}}$ and $v_{*_{2}}$ are the distinct vertices of edge $E_{i j}$. 


$$
\text { 3. } W V_{*}=P \cap\left(\bigcap_{s, t \in * ; V . \subset E_{s t}} C_{s t *}^{+}\right) \text {. }
$$

Proof of Wedge Representation Theorem: We provide the proof for the edge wedge representation. A similar argument holds for the face and vertex wedge representations.

2. Let $\overline{p q} \subset W E_{i j}$ where $\overline{p q}$ is a normal chord of $E_{i j}$ with $p \in E_{i j}$. Then $p, q \in P$ since $W E_{i j} \subset P$ by definition. Let $S$ be the supporting hyperplane of $P$ containing $E_{i j}$ such that $\overline{p q} \perp S$. We need to show that $q$ is on the same side of $H_{i j}$ as $F_{i}$ and $q$ is on the same side of $H_{j i}$ as $F_{j}$. If not, then without loss of generality $\overline{p q}$ is on the side opposite to $F_{i}$ from $H_{i j}$. Therefore, $S$ intersects the relative interior of $P$, which is a contradiction. So, $q$ is on the same side of $H_{i j}$ as $F_{i}$ and $q$ is on the same side of $H_{j i}$ as $F_{j}$. Also, $\overline{p q}$ is on the same side as $E_{i j}$ for each $C_{i j *}$ since $\overline{p q} \| C_{i j *}$ or $\overline{p q} \subset C_{i j *}$ and $p \in E_{i j}$. Therefore, $\overline{p q} \subset P \cap H_{i j}^{+} \cap H_{j i}^{+} \cap C_{i j *_{1}}^{+} \cap C_{i j *_{2}}^{+}$

Let $r \in P \cap H_{i j}^{+} \cap H_{j i}^{+} \cap C_{i j *_{1}}^{+} \cap C_{i j *_{2}}^{+}$where $V_{*_{1}}$ and $V_{*_{2}}$ are the distinct vertices of edge $E_{i j}$. Let $p$ be the point in $\operatorname{aff}\left(E_{i j}\right)$ such that $\overline{p r} \perp \operatorname{aff}\left(E_{i j}\right)$. Then $p \in E_{i j}$ since $r$ is on the same side of $C_{i j *}$ as $E_{i j}$ and the $C_{i j *}^{\prime} s$ are perpendicular to $E_{i j}$. Let $S$ be the plane perpendicular to $\overline{p r}$. The angle between $\overline{p r}$ and $F_{i}$ is less than $\pi / 2$ since $C$ lies in the same halfspace as $F_{i}$ of $H_{i j}$. Therefore $r$ and $F_{i}$ lie on the same side of $S$. Similarly, $r$ and $F_{j}$ lie on the same side of $S$. Since $E_{i j} \in S$ and all of the facets which contain $E_{i j}$ are in the same half space of $S$ then $P$ is contained in a halfspace of $S$. So, $S$ is a supporting hyperplane of $P$ and therefore $r$ is in $W E_{i j}$.

We now define the molecules, which will be used to partition the complements of the wedges.

DEFINITION. Given a face $F_{i}$ and edge $E_{i j}$, the face molecule associated with an edge is $F_{i} E_{i j}=\left(P-W F_{i}\right) \cap H_{i j}^{-} \cap C_{i j *_{1}}^{+} \cap C_{i j *_{2}}^{+}$where $V_{*_{1}}$ and $V_{*_{2}}$ are the distinct vertices of edge $E_{i j}$.

Definition. Given a face $F_{i}$ and one of its incident vertices $V_{*}$, the face molecule associated with a vertex is $F_{i} V_{*}=\left(P-W F_{i}\right) \cap C_{i j_{*}}^{-} \cap C_{i k *}^{-}$where $V_{*}$ is contained in edges $E_{i j}$ and $E_{i k}$ such that $E_{i j}, E_{i k} \subset F_{i}$.

DEFINITION. Given an edge $E_{i j}$ and face $F_{i}$, the edge molecule associated with a face is $E_{i j} F_{i}=\left(P-W E_{i j}\right) \cap H_{i j}^{-} \cap C_{i j *_{1}}^{+} \cap C_{i j *_{2}}^{+}$where $V_{*_{1}}, V_{*_{2}} \subset E_{i j}$.

DEFINITION. Given an edge $E_{i j}$ and one of its incident vertices $V_{*}$, the edge molecule associated with a vertex is $E_{i j} V_{*}=\left(P-W E_{i j}\right) \cap C_{i j *}^{-}$.

DEFINITION. Given a vertex $V_{*}$ which is contained in a face $F_{i}$, the vertex molecule associated with a face is $V_{*} F_{i}=\left(P-W V_{*}\right) \cap C_{i j *}^{-} \cap C_{i l *}^{-}$where $V_{*} \subset E_{i j}, E_{i l} \subset F_{i}$.

DeFInITION. Given a vertex $V_{*}$ which is contained in an edge $E_{i j}$, the vertex molecule associated with an edge is $V_{*} E_{i j}=\left(P-W V_{*}\right) \cap C_{i j *}^{-} \cap C_{i k_{*}}^{+} \cap C_{j l *}^{+}$where $E_{i k}$ is the unique edge such that $V_{*} \subset E_{i k} \subset F_{i}$ and $E_{i k} \neq E_{i j}$. Similarly, $E_{j l}$ is the unique edge such that $V_{*} \subset E_{j l} \subset F_{j}$ and $E_{j l} \neq E_{i j}$. 
Pictured below in Figure 3 is an edge wedge and its associated molecules for the polytope $Q$ (see Figure 2 for labeling). Polytope 1 is $W E_{12}$. Polytope 3 is an edge molecule associated with a vertex and polytopes 2 and 4 are edge molecules associated with faces. Polytope 3 is $E_{12} V_{\{1,2,3\}}$, polytope 2 is $E_{12} F_{1}$ and polytope 4 is $E_{12} F_{2}$.

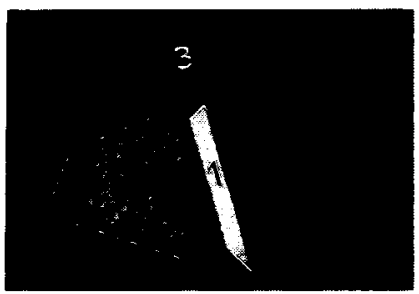

Figure 3. An edge wedge and its associated molecules.

Figure 4 below illustrates a vertex wedge and its associated molecules for the polytope $Q$. Polytope 1 is $W V_{\{1,2,3\}}$. Polytopes 3,5 and 7 are vertex molecules associated with edges and polytopes 2,4 and 6 are vertex molecules associated with faces. Polytope 2 is $V_{\{1,2,3\}} F_{3}$, polytope 3 is $V_{\{1,2,3\}} E_{1,3}$, polyotpe 4 is $V_{\{1,2,3\}} F_{1}$, polytope 5 is $V_{\{1,2,3\}} E_{12}$, polytope 6 is $V_{\{1,2,3\}} F_{2}$, and polytope 7 is $V_{\{1,2,3\}} E_{2,3}$.

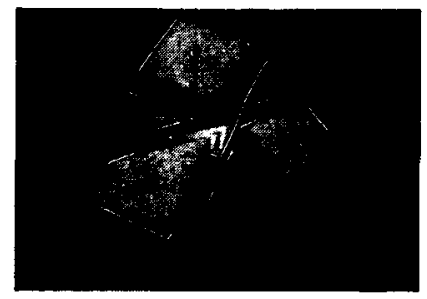

Figure 4. A vertex wedge and its associated molecules.

Redundancy Lemma. In the six definitions above, the complement of a wedge $(P-$ wedge $)$ can be replaced by $P$.

Proof: In each case, except $F_{i} V_{*}$, this follows immediately by comparing the wedges in the Wedge Representation Theorem above, with the definitions of the various molecules. For the case $F_{i} V_{*}$, the hyperplane $H_{i j}$ "separates" $W F_{i}$ from $\left(C_{i j *}^{-} \cap C_{i k *}^{-}\right)$. Therefore, $\left(C_{i j_{*}}^{-} \cap C_{i k_{*}}^{-}\right) \cap P \subseteq P-W F_{i}$. Hence $\left(P-W F_{i}\right)$ can be replaced by $P$.

The following lemma is crucial in the proof of the Identical Molecule Theorem found in Section 5 which is subsequently used in the proof of the Wedge Complement Theorem.

SYMMETRY LEMMA $E_{i j} F_{i}=F_{i} E_{i j}$ and $V_{*} F_{i}=F_{i} V_{*}$.

Proof: The proof is trivial by applying the Redundancy Lemma to the definitions of the relevant molecules.

For an illustration of the symmetry lemma for polytope $Q$ refer back to Figures 1 , 3 and 4 . Polytope 2 in Figure $1\left(F_{1} E_{12}\right)$ equals polytope 2 in Figure $3\left(E_{12} F_{1}\right)$. Also, 
polytope 3 in Figure $1\left(F_{1} V_{\{1,2,3\}}\right)$ equals polytope 4 in Figure $4\left(V_{\{1,2,3\}} F_{1}\right)$. Next we use the Wedge Representation Theorem and the definitions of the molecules in order to partition the complements of the wedges.

\section{Partition Theorems}

In this section, we use the molecules to define partitions of the face, edge and vertex wedge complements. The face and edge partition theorems state that the wedge complements are partitioned by the face and edge molecules. It is also the case that the vertex wedge complement is the union of the vertex molecules, though this union is not necessarily disjoint, and thus these molecules do not give us a partition. Since the vertex molecules associated with faces may have a non-trivial intersection, we subtract these intersections where needed, thereby creating a partition. We provide the proof of the Vertex Partition Theorem below. The Face and Edge Partition Theorems follow from convexity and properties of normals, and therefore can be verified by the reader.

FACE Partition Theorem Given a face $F_{i}$ the face wedge complement is partitioned by all of the face molecules associated with the vertices and edges of that face.

$$
P-W F_{i}=\sum_{s=1}^{f_{0}\left(F_{i}\right)} F_{i} V_{*_{s}}+\sum_{t=1}^{f_{1}\left(F_{i}\right)} F_{i} E_{i j_{t}} .
$$

EDge Partition Theorem. Given an edge $E_{i j}$ the edge wedge complement is partitioned by all of the edge molecules associated with the vertices and faces incident to that edge.

$$
P-W E_{i j}=E_{i j} F_{i}+E_{i j} F_{j}+E_{i j} V_{* 1}+E_{i j} V_{* 2}
$$

where $i, j \in *_{1}, *_{2}$.

Next we prove the Vertex Partition Theorem. This proof is more complicated than the proofs of the previous two theorems. The proof entails choosing a vertex and relabelling its incident edges and faces. The proof then establishes set equality and disjointness.

Vertex Partition Theorem. Given a vertex $V_{*}$ the vertex wedge complement is the union of all of the vertex molecules associated with the faces and edges incident to that vertex. Moreover,

$$
P-W V_{*}=\sum_{x \in *}\left(V_{*} F_{x}-V_{*} I_{x y}\right)+\sum_{s, t \in * ; V_{*} \subset E_{s t}} V_{*} E_{s t},
$$

where $V_{*} I_{x y}=V_{*} F_{x} \cap V_{*} F_{y}$ and the index $y$ comes from the face $F_{y}$, containing $V_{*}$, which is the face to the "left" of $F_{x}$ with respect to the vertex $V *$ as described below in the proof. 
Proof of Vertex Partition Theorem: In the proof of this theorem we shall allow the temporary relabelling of a vertex and its incident edges. Given a vertex $\nu$ label its incident edges $\varepsilon_{1}, \ldots, \varepsilon_{n}$ where these edges are ordered such that $\varepsilon_{i}$ and $\varepsilon_{i+1}$ share a face and such that from the position of an outward normal emanating from $\nu$, the labelling is counter-clockwise. In the same manner, relabel the faces $\phi_{1}, \ldots, \phi_{n}$ such that $\phi_{i}$ is to the "right" of $\varepsilon_{i}$, so that $\phi_{i} \cap \phi_{i+1}=\varepsilon_{i}$. All arithmetic for the indices in this proof is modulo $n$. Hence, $\varepsilon_{n+1}=\varepsilon_{1}$. Note that with this relabelling, we need to prove

$$
P-W \nu=\sum_{i=1}^{n}\left(\nu \phi_{i}-\nu I_{i(i+1)}\right)+\sum_{i=1}^{n} \nu \varepsilon_{i}
$$

Each edge $\varepsilon_{i}$ is associated with a cutting plane $C_{i}$, perpendicular to $\varepsilon_{i}$ and containing vertex $\nu$. Note that $C_{n+1}=C_{1}$ and $C_{0}=C_{n}$. If $p$ is in the same half-space as $\varepsilon_{i}$, then $p \in C_{i}^{+}$. If $p$ is in the opposite half-space as $\varepsilon_{i}$, then $p \in C_{i}^{-}$. Note that with the new notation,

$$
\begin{aligned}
\nu \varepsilon_{i} & =(P-W \nu) \cap C_{i-1}^{+} \cap C_{i}^{-} \cap C_{i+1}^{+} \\
\nu \phi_{i} & =(P-W \nu) \cap C_{i-1}^{-} \cap C_{i}^{-} \\
\nu I_{i(i+1)} & =(P-W \nu) \cap C_{i-1}^{-} \cap C_{i}^{-} \cap C_{i+1}^{-} \\
\nu \phi_{i}-\nu I_{i(i+1)} & =(P-W \nu) \cap C_{i-1}^{-} \cap C_{i}^{-} \cap C_{i+1}^{+} .
\end{aligned}
$$

PROOF OF SET EQUALITY: Since each $\nu \phi_{i}$ and $\nu \varepsilon_{i}$ are by definition a subset of $P-W \nu$, we have $\sum_{i=1}^{n}\left(\nu \phi_{i}-\nu I_{i(i+1)}\right)+\sum_{i=1}^{n} \nu \varepsilon_{i} \subseteq P-W \nu$. Next we show containment in the other direction.

Let $p$ be a point in $P-W \nu$. Consider each $C_{i}$ and its corresponding edge $\varepsilon_{i}$. If $p \in C_{i}^{+}$, for all $i$, then $p \in W \nu$. Otherwise, there is at least one $C_{i}$ such that $p \in C_{i}^{-}$. CASE 1. $p \in C_{i-1}^{+}$and $p \in C_{i+1}^{+}$. Then $p \in \nu \varepsilon_{i}$ by the definition of a vertex molecule associated with an edge.

CASE 2. $p \in C_{i-1}^{-}$or $p \in C_{i+1}^{-}$. Assume $p \in C_{i-1}^{-}$, (the same argument is valid for $p \in C_{i+1}^{-}$). Then $p \in \nu \phi_{i}$ by the definition of a vertex molecule associated with a face. The point $p$ may also be in the vertex molecule $\nu \phi_{i+1}$ and hence $p \notin \nu \phi_{i}-\nu I_{i(i+1)}$. In fact, $p$ is contained in a consecutive chain of vertex molecules

$$
p \in \nu \phi_{i} \cap \nu \phi_{i+1} \cap \ldots \cap \nu \phi_{i+m}
$$

where this chain may have length 1 and must have length less than $n$. If the chain were to have length $n$, then $p \in C_{i}^{-}$, for all $i$. This means that for each of these edges $\varepsilon_{i}$, the point on the edge closest to $p$ is $\nu$. Consider the plane $G$ through $\nu$ perpendicular to $\overline{\nu p}$. Since $\nu$ is the closest point to $p$, all of the edges $\varepsilon_{i}$ lie in the closed half-space bounded by $G$ which does not contain $p$. Since all of the edges for this vertex lie on one side of 
the hyperplane $G$, then the polytope $P$ lies on on'e side of $G$ and hence $G$ is a supporting hyperplane of $P$. Therefore, $p \notin P$. This is a contradiction, therefore this chain has length less than $n$.

By definition of this chain, $p \notin \nu \phi_{i+m+1}$. Therefore $p \in \nu \phi_{i+m}-\nu I_{(i+m)(i+m+1)}$. This establishes set equality.

PRoOf THAT THE COMPONENTS ARE DISJOINT: Consider $p \in P-W \nu$ and the vertex $\nu$. Let $G$ be the hyperplane through $\nu$ perpendicular to the chord $\overline{\nu p}$. Let $G^{+}$be the half-space containing $p$ and $G^{-}$, the other half-space. Since each $\varepsilon_{i}$ contains $\nu$, each $\varepsilon_{i} \subset G^{+}, G^{-}$or $G$. If an $\varepsilon_{j}$ lies in $G$, then the point $p$ lies in the hyperplane $C_{j}$ which is a set of measure zero and therefore can be disregarded in this proof. The hyperplane $G$ divides the set of ordered edges $\varepsilon_{1}, \varepsilon_{2}, \ldots, \varepsilon_{n}$ into two contiguous subsets modulo $n$. If $\varepsilon_{i}$ is in $G^{-}$, then $\nu$ is the closest point to $p$ on $\varepsilon_{i}$ and thus $p \in C_{i}^{-}$. If $\varepsilon_{i}$ is in $G^{+}$, then the point on the edge closest to $p$ is not $\nu$ and thus $p \in C_{i}^{+}$.

By combining the two above ideas, we have

$$
p \in C_{s}^{-} \cap C_{s+1}^{-} \cap \ldots \cap C_{s+t}^{-} \cap C_{s+t+1}^{+} \cap C_{s+t+2}^{+} \cap \ldots \cap C_{s+t+m}^{+}
$$

where $1 \leqslant s \leqslant n, 0 \leqslant t \leqslant n-2,0 \leqslant m \leqslant n-1, t+m+1=0$ modulo $n$.

CASE 1. If $p \in \nu \varepsilon_{i}$ then $p \notin \nu \varepsilon_{s}$, for all $s \neq i$ and $p \notin \nu \phi_{j}-\nu I_{j(j+1)}$, for all $j$.

Since $p \in \nu \varepsilon_{i}, p \in(P-W \nu) \cap C_{i-1}^{+} \cap C_{i}^{-} \cap C_{i+1}^{+}$. Because the C-hyperplanes which are adjacent to $C_{i}^{-}$in the chain are both labelled "+", this means that $C_{i}^{-}$is the only C-hyperplane in the chain which is labelled with a "-". Since there are no other $C^{-}$ in the chain, $p$ can not be contained in any other $\nu \varepsilon_{s}$. Likewise, since there are no two $C_{j-1}^{-}, C_{j}^{-}$in the chain, $p$ can not be contained in any $\nu \phi_{j}-\nu I_{j(j+1)}$.

CASE 2: If $p \in \nu \phi_{i}-\nu I_{i(i+1)}$ then $p \notin \nu \varepsilon_{s}$, for all $s$ and $p \notin \nu \phi_{j}-\nu I_{j(j+1)}$, for all $j \neq i$.

Since $p \in \nu \phi_{i}-\nu I_{i(i+1)}, p \in(P-W \nu) \cap C_{i-1}^{-} \cap C_{i}^{-} \cap C_{i+1}^{+}$. Since there are at least two C-hyperplanes which are labelled "-", in the chain, there are no sequences which are labelled ",,+-+ ". Therefore, $p \notin \nu \varepsilon_{s}$, for all $s$. In the chain of C-hyperplanes, there is only one sequence labelled ",,--+ " (when the index is considered in increasing order, modulo n). Therefore, $p \notin \nu \phi_{j}-\nu I_{j(j+1)}$, for all $j \neq i$.

Therefore, the components in the union are disjoint.

These three theorems partition the wedge complements and set the stage for proving the Wedge Complement Theorem.

\section{Proof of the Wedge Complement Theorem}

In order to prove the Wedge Complement Theorem, WCT, we shall establish a one to one correspondence between the edge molecules and particular combinations of the vertex and face molecules. This correspondence will actually relate equal portions of the polytope. Each edge molecule associated with a face will be matched with a face 
molecule associated with an edge. The correspondence for the edge molecules associated with vertices is more complicated. Each edge molecule associated with a vertex will be matched with a combination of vertex molecules associated with faces, vertex molecules associated with edges and face molecules associated with vertices. We begin by defining the correspondence between the molecules and proving that it is one to one and onto. We then show that this correspondence relates equal pieces in the Correspondence Identity Theorem. Finally, we combine these two theorems with the face, vertex, and edge partition theorems to give the proof of the Wedge Complement Theorem.

Definition. Given a polytope $P$, define the molecule correspondence between the set of all edge molecules and the set containing all vertex and face molecules by

$$
\begin{aligned}
E_{i j} F_{i} \leftrightarrow F_{i} E_{i j} \\
E_{i j} V_{*} \leftrightarrow V_{*} E_{i j} \cup\left(V_{*} F_{s}-V_{*} I_{i j}\right) \cup F_{t} V_{*}
\end{aligned}
$$

This correspondence is well defined, where $s$ and $t$ are chosen as follows. From the position of an outward normal emanating from a vertex $V_{*}$, there is a unique face $F_{s}$ to the right of $E_{i j}$. Note that $s$ is one of the two indices $i$ or $j$. Let $t$ be the other of these two indices.

Clearly, every $E F, F E, E V$ and $V E$ appear once and only once in the correspondence. Every $V F$ appears in the correspondence exactly once, since given a vertex $V$ and face $F$, that face is to the right of exactly one edge containing $V$. Similarly, every $F V$ appears in the correspondence exactly once, since given a vertex $V$ and face $F$, that face is to the left of exactly one edge containing $V$. Therefore the molecule correspondence is one to one and onto.

CoRrespondence IDENTITY THEOREM. The molecule correspondence associates identical portions of $P$ arising from different partitions of $P$.

Proof: We have $E_{i j} F_{i}=F_{i} E_{i j}$ by the Symmetry Lemma found in Section 3.

It remains to be shown that $E_{i j} V_{*}=V_{*} E_{i j} \cup\left(V_{*} F_{s}-V_{*} I_{i j}\right) \cup F_{t} V_{*}$. Without loss of generality, assume that $E_{i j} V_{*} \leftrightarrow V_{*} E_{i j} \cup\left(V_{*} F_{i}-V_{*} I_{i j}\right) \cup F_{j} V_{*}$. Note that $\left(V_{*} F_{i}-V_{*} I_{i j}\right) \cup$ $F_{j} V_{*}=V_{*} F_{i} \cup F_{j} V_{*}$ since $F_{j} V_{*}=V_{*} F_{j}$ and $V_{*} I_{i j}=F_{i} V_{*} \cap F_{j} V_{*}$. Therefore, it suffices to prove that $E_{i j} V_{*}=V_{*} E_{i j} \cup V_{*} F_{i} \cup F_{j} V_{*}$.

If $p \in V_{*} E_{i j} \cup V_{*} F_{i} \cup F_{j} V_{*}$, then $p \in C_{i j *}^{-}$and hence $p \in E_{i j} V_{*}$.

Consider $p \in E_{i j} V_{*}=P \cap C_{i j_{*}}^{-}$. Given any hyperplane $C, p \in C^{+}$or $p \in C^{-}$. Let the relevant cutting hyperplanes be $C_{i j *}, C_{i k *}$ and $C_{j l *}$. Since $p \in C_{i j *}^{-}$, then $p$ is in one of the following four sets:

1. $C_{i j *}^{-} \cap C_{i k *}^{+} \cap C_{j i *}^{+}$

2. $C_{i j *}^{-} \cap C_{i k *}^{+} \cap C_{j i *}^{-}$

3. $C_{i j *}^{-} \cap C_{i k *}^{-} \cap C_{j k *}^{+}$

4. $C_{i j *}^{-} \cap C_{i k *}^{-} \cap C_{j l *}^{-}$. 
In Case 1, $p \in V_{*} E_{i j}$. In Cases 2 and $4, p \in F_{j} V_{*}$. In Case 3,p $\in V_{*} F_{i}$. Hence, $p \in V_{*} E_{i j} \cup V_{*} F_{i} \cup F_{j} V_{*}$.

Therefore $E_{i j} V_{*}=V_{*} E_{i j} \cup\left(V_{*} F_{i \text { or } j}-V_{*} I_{i j}\right) \cup F_{j \text { or } i} V_{*}$ and hence have the same volume.

Below are two examples of corresponding molecules for polytope $Q$. Consider $E_{12} F_{1}=F_{1} E_{12}$. The molecule $F_{1} E_{12}$, labelled 2 in Figure 1, corresponds with the molecule $E_{12} F_{1}$, also labelled 2 in Figure 3. Next consider $E_{12} V_{\{1,2,3\}}=V_{\{1,2,3\}} E_{12}$ $\cup\left(V_{\{1,2,3\}} F_{2}-V_{\{1,2,3\}} I_{12}\right) \cup F_{1} V_{\{1,2,3\}}$. The molecules in this equation can be found in the following figures: $E_{12} V_{\{1,2,3\}}$ is labelled 3 in Figure 3, $V_{\{1,2,3\}} E_{12}$ is labelled 5 in Figure $4, V_{\{1,2,3\}} F_{2}$ is labelled 6 in Figure 4 and $F_{1} V_{\{1,2,3\}}$ is labelled 3 in Figure 1.

Note that $V_{\{1,2,3\}} I_{12}=\emptyset$ since $V_{\{1,2,3\}} F_{1} \cap V_{\{1,2,3\}} F_{2}=\emptyset$ as seen in Figure 4. So far we have dissected the wedge complements and matched equal polytopes. This matching connects wedge complements of edge wedges with wedge complements of vertex and face wedges. We are now ready to prove the Wedge Complement Theorem which completes the proof of the Euler 7 Volume Theorem.

Wedge Complement Theorem. Given a convex polytope $P$ in $\mathbb{R}^{3}$ the sum of the face wedge complements plus the sum of the vertex wedge complements is congruent by addition to the sum of the edge wedge complements,

$$
\sum_{i=1}^{f}\left(P-W F_{i}\right)+\sum_{i=1}^{v}\left(P-W V_{i}\right) \stackrel{ \pm}{\cong} \sum_{i=1}^{e}\left(P-W E_{i}\right)
$$

Proof of Wedge Complement Theorem: By the Edge Partition Theorem found in Section 4, we have for each edge $E_{i j}$,

$$
\left(P-W E_{i j}\right)=E_{i j} F_{i}+E_{i j} F_{j}+\sum_{V_{*} \subset E_{i j}} E_{i j} V_{*}
$$

So,

$$
\begin{aligned}
\sum_{\text {all edges } E_{i j}}\left(P-W E_{i j}\right) & =\sum_{\text {all edges } E_{i j}} \sum_{V \cdot \subset E_{i j}} E_{i j} V_{*} \\
& +\sum_{\text {all edges } E_{i j}}\left(E_{i j} F_{i}+E_{i j} F_{j}\right) .
\end{aligned}
$$

The Molecule Correspondence and Correspondence Identity Theorem give us

$$
\sum_{\text {all edges } E_{i j}}\left(E_{i j} F_{i}+E_{i j} F_{j}\right)=\sum_{\text {all faces } F_{i}} \sum_{E_{i j} \subset F_{i}} F_{i} E_{i j}
$$


and

$$
\begin{aligned}
\sum_{\text {all edges } E_{i j}}\left(\sum_{V \cdot \subset E_{i j}} E_{i j} V_{*}\right) & =\sum_{\text {all vertices } V_{*}} \sum_{i j \in *} V_{*} E_{i j} \\
& +\sum_{\text {all vertices } V_{*}} \sum_{F_{i} \supset V_{*}}\left(V_{*} F_{i}-V_{*} I_{i j}\right) \\
& +\sum_{\text {all faces } F_{i}} \sum_{V_{*} \subset F_{i}} F_{i} V_{*}
\end{aligned}
$$

Substituting equations (2) and (3) into equation (1), we obtain,

$$
\begin{aligned}
\sum_{\text {all edges } E_{i j}}\left(P-W E_{i j}\right) & =\sum_{\text {all vertices } V_{*}} \sum_{i j \in *} V_{*} E_{i j} \\
& +\sum_{\text {all vertices } V_{*}} \sum_{F_{i} \supset V_{*}}\left(V_{*} F_{i}-V_{*} I_{i j}\right) \\
& +\sum_{\text {all } F_{i}} \sum_{V_{*} \subset F_{i}} F_{i} V_{*} \\
& +\sum_{\text {all faces } F_{i}} \sum_{E_{i j} \subset F_{i}} F_{i} E_{i j} .
\end{aligned}
$$

Applying the Face and Vertex Partition theorems, we obtain,

$$
\sum_{\text {all faces } F_{i}} P-W F_{i}=\sum_{\text {all faces } F_{i}}\left(\sum_{E_{i j} \subset F_{i}} F_{i} E_{i j}+\sum_{V \cdot \subset F_{i}} F_{i} V_{*}\right)
$$

and

$$
\sum_{\text {all vertices } V_{0}}\left(P-W V_{*}\right)=\sum_{\text {all vertices } V .}\left(\sum_{i, j \in *} V_{*} E_{i j}+\sum_{F_{i} \supset V_{*}}\left(V_{*} F_{i}-V_{*} I_{i j}\right)\right) .
$$

Combining equations (4), (5) and (6), we obtain,

$$
\sum_{\text {all edges } E_{i j}}\left(P-W E_{i j}\right) \stackrel{ \pm}{\cong} \sum_{\text {all faces } F_{i}}\left(P-W F_{i}\right)+\sum_{\text {all vertices } V_{*}}\left(P-W V_{*}\right),
$$

which completes the proof.

\section{REFERENCES}

[1] H. Eves, A survey of geometry (Allyn and Bacon, Inc., Boston, 1972).

[2] K. Hann, 'What's the bound on the average number of normals', Amer. Math. Monthly 103 (1996), 897-900.

[3] K. Hann, 'The average number of normals through a point in a convex body and a related Euler-type identity', Geom. Dedicata 48 (1993), 27-55.

[4] D. Hug, 'On the mean number of normals through a point in the interior of a convex body', Geom. Dedicata 55 (1995), 319-340. 
Department of Mathematics and Computer Science California State University

Hayward CA 94542

United States of America

e-mail: callahan@csuhayward.edu

khann@csuhayward.edu 